\title{
Histopathologic Evaluation of the Subcutaneous Tissue Response toThree Endodontic Sealers
}

\author{
Dr. Mini.K.John \\ Assistant Professor,Department of Conservative and Endodontics, Government Dental College, \\ Trivandrum,Kerala,India
}

\begin{abstract}
The materials used for obturation of root canal system may get extruded through apical foramen into the per apical tissue. Therefore, biocompatibility of these materials is very important. The purpose of this study was to evaluate the in vivo biocompatibility of three endodontic sealers: Sealapex, Diaket and Tubliseal after their subcutaneous implantation in rats. Each of the materials was injected subcutaneously in the dorsal connective tissue of 20 Wistar albino rats. Tissue biopsies were collected at first day, fifth day, tenth day and thirtieth day after the procedure. The specimens were processed and stained with hematoxylin and eosin and examined microscopically. According to this study, all of the sealers cause inflammatory reactions immediately after contact with tissue, but the intensity of these responses decrease with time. The acute inflammatory responses of initial days changed to chronic proliferative, phase and later to healing processes by the end of one month.
\end{abstract}

Key words: Endodontic Sealers, Tissue reaction (response), Subcutaneous tissue reaction.

\section{Introduction}

The materials used for obturation of root canal system such as sealers, may get extruded through apical foramen into the periapical tissue. Therefore, the biocompatibility of these materials is very important. Currently, there are three recommended tests for the biological evaluation and acceptance of endodontic materials: a primary test or cell culture test which provides a general profile of toxicity for the material (level 1), a secondary test or material implantation test which evaluates its local toxicity in experimental animals (level 2) and usage test in which the material is used in the endodontic treatment of teeth in experimental animals (level $3)$.

In cell culture tests, several studies have been performed and all have found different degrees of cytotoxicity for various sealers [1, 2,3]. Bouillaguet et al (2004)[1] evaluated cytotoxicity of several sealers at cell culture and reported that the cytotoxicity of sealers increases with time from $24 \mathrm{hr}$ to 1 week and most sealers are potentially, cytotoxic specially when they are mixed freshly . Huangetal [3] demonstrated that Diaket exhibit not only in vitro dose dependent cytotoxicity but also genotoxicity and that cytotoxicity of ZOE was detectable as early as $1 \mathrm{hr}$ after mixing and remained at a high level until 5 week. Diaket, however, induced early cytotoxic effects that lasted for 1 week, followed by a substantial reduction in cytotoxicity [8]. In usage tests, Bernath and Szabo has found that after filling the root canals of monkeys, all sealers cause inflammatory response and reported that if root filling by Apexit and Grossman's sealers confine to the canal system, it would not cause inflammation. But similar situations with Diaket, cause mild lymphocytic- plasmocytic infiltration in some cases .The reports about biocompatibility of sealers are different. Thus in present study the biocompatibility of three conventional sealers such as Diaket, Sealapex, and Tubliseal are evaluated by subcutaneous injection (secondary test) in rats.

\section{Materials and methods}

Twenty mature male Albino rats, weighing from 250 to $500 \mathrm{gms}$, were used in this study. . Animal care was carried out according to the Institutional Animal Care and Use Committee .Animals were divided into four group with 5 animals in each group to avoid bias .Every 5 animal in each group were sacrificed at the end of time interval of one day, five days ,ten days and thirty days. The animals were anesthetized with an intraperitoneal injection of $65 \mathrm{mg} / \mathrm{ml}$ sodium pentobarbital at a dose of $5.1 \mathrm{mg} / 100 \mathrm{~g}$ body weight. ).

After disinfection of skin, the dorsum was shaved at 4 points, two points at anterior or cranial portion (right and left) and two points at posterior or caudal portion (right and left).The materials were freshly mixed in accordance with the manufacturer's instruction. All rats were injected with $.05 \mathrm{ml}$ of each of the three sealers and distilled water at four predesignated sites which were specifically encircled. Once procedure was completed each group of animals were caged in separate cages. Post-operatively local examination of sites was done for detection of infection.

The study was conducted in four parts; the rats were sacrificed at different time intervals after injecting the test sealers, five rats after one day (Group A), five rats after 5 days (Group B), five rats after 10 days and another five after 30 days (Group D) by anesthetic over dosage. Biopsy (specimen and $2 \mathrm{~mm}$ of surrounding 
normal tissue) of the injection sites were taken. Macroscopically examined for inflammation or any other abnormalities and placed in $10 \%$ formalin. The tissue was processed to be embedded in paraffin after 48 hrs of fixation. The blocks were cut to thickness of 6 micrometer. The sections were mounted on glass slides and were stained with haematoxylin and eosin. The histopathological evaluations of specimens were performed by pathologist under light microscope.

For each material, the sum and average of inflammatory cells (polymorphonuclear leukocytes (PMNs), plasma cells, lymphocytes, macrophages and giant cells) and fibroblasts were determined in ten separate areas at 400x magnification. The observer was blinded to the tissue source. Reactions in the tissue were scored as: 0 , none or few inflammatory cells (no reaction); 1, less than 25 cells (mild reaction); 2, between 25 and 125 cells (moderate reaction); and 3, 125 or more cells (severe reaction) Results were statistically analyzed using the Kruskal-Wallis and Wilcoxin Signed rank test

\subsection{Sealapex}

\section{Materials}

A severe reaction was observed on the $1^{\text {st }}$ day .The tissue was disorganized and infiltrated with neutrophils, but there were no giant cells or areas of necrosis (Figures 1A ). On the 5th day, the tissue was more organized and was characterized by the presence of chronic cells and absence of fibrous capsule formation and areas of necrosis (Figures 1B). The intensity of the reaction was milder on the 10th day (Figures1C). At $30^{\text {th }}$ day moderate amount of chronic inflammatory cells with mild amount of fibrosis was seen (Figure 1D)

\subsection{Tubliseal}

A severe inflammatory reaction with some edematous tissue was seen on the $1^{\text {st }}$ day. The tissue was infiltrated with neutrophils and few macrophages. There were no giant cells or areas of necrosis (Figures 2A ). The intensity of the inflammatory reaction was milder on the $5^{\text {th }}$ day and the tissue was more organized exhibiting the formation of connective fibers. The tissue was infiltrated with macrophages, plasma cells and lymphocytes. There were no giant cells or area of necrosis and organization of a fibrous capsule was observed in this period of time (Figures $2 \mathrm{~B}$ ). On the $10^{\text {th }}$ day, granulation tissue with muscle necrosis was seen( Figure $2 \mathrm{C}$ ). There inflammatory reaction was more severe on the $30^{\text {th }}$ day characterized by presence of chronic cells including giant cells (Figures 2D).

\subsection{Diaket}

Mild acute inflammation without areas of edema was observed on the $1^{\text {st }}$ day. The inflammatory reaction was characterized by presence of neutrophil (Figures 3A). The intensity of the reaction was attenuated at the 5th day and chronic inflammatory cells were predominantly observed. The tissue was in an initial state of organization with presence of few fibroblasts and connective fibers (Figures 3B). On the $10^{\text {th }}$ day, connective tissue with fibers and few fibroblasts was observed and a fibrous capsule tissue was present. Macrophages and giant cells with material in their cytoplasm were also observed (Figures $3 \mathrm{C}$ ). On $30^{\text {th }}$ day chronic inflammatory reaction with mild amount of muscle necrosis and moderate amount of fibrosis and foreign body reaction was seen. Tissue reaction showed a transition stage to develop into granuloma (Figure 3D).

\subsection{Distilled water}

On the $1^{\text {st }}$ day mild inflammatory reaction with tissue distension was observed (Figure $4 \mathrm{~A}$ ), by $30^{\text {th }}$ day inflammatory reaction subsided completely (Figure 4D)

\subsection{Day 1}

\section{Evaluation Periods}

All the inflammatory cells except for oedema have significant association with the material used. Oedema,could more be due to the surgical trauma than due to injection of material and is found more with the Tubliseal and the variation is statistically significant at 0.05 level(Table 1).Comparison of acute inflammation at day 1 based on the material used is given in Table1. Severity of PMNLs is more in Sealapex (3 severe and1 moderate, mean score 2.2), followed by Tubliseal (mean score 1.4) and least in Distilled water (4 mild, mean score $=0.8)$. The Kruskal Wallis Test $(\mathrm{p}>0.05)$ shows that the variation in severity of PMNLs among the four materials is not statistically significant (Table 2).

The severity of eosinophils is also found slightly high among Sealapex, but the variation of severity in eosinophils found in the materials is not statistically significant $(\mathrm{p}>0.05)$.

When considering the severity in lymphocytes, it is high (mean=1.2) in Sealapex (4 mild and 1 moderate) and no inflammation found among Tubliseal and I - mild inflammation found in Diaket and Distilled water. The Kruskal Wallis Test $(\mathrm{p}<0.01)$ shows that the material used is significantly associated with the level 
of inflammation in lymphocytes. The pair wise comparison shows that the inflammation in lymphocytes is significantly high in Sealapex when compared with all the other three materials $(p<0.05)$.

\subsection{Day 5}

The distribution of the scores attributed to the materials is given on Table 3. None of the neither acute nor chronic inflammatory cells showed any significance in relation to the experimental materials. Edema is the only significant tissue reaction during this period $(\mathrm{p}<.05)$, (Table 4) was more in relation to Diaket than Tubliseal and was insignificant $(\mathrm{p}>.05)$ in relation to other two test materials.

\subsection{Day 10}

The distribution of the scores attributed to the materials is given on Table 5.significant amount of lymphocytes were seen in association with all the test materials $(\mathrm{p}=.014)$. In all test materials including control, statistically significant $(\mathrm{p}<.05)$ amount of lymphocytes were found. The lymphocytic proliferation was seen highest in case of Diaket when compared to other two materials (Table 6).Edema is still persistent and evident in this time period with all materials and it is most severe with Diaket and Tuliseal $(p<.05)$. Another tissue reaction that is significant is muscle necrosis $(\mathrm{p}=.013)$, it is relatively more in Sealapex $(\mathrm{p}<.05)$ than in other two test samples.

\subsection{Day 30}

At $30^{\text {th }}$ day both acute and chronic cells is seen to negligible level and tissue has regained its normal health or healing has started (Table 7). Only tissue reaction that is evident is muscle necrosis ( $p=.018)$ Muscle necrosis is significant in Diaket and Tubliseal $(\mathrm{p}<.05)$.

\section{Discussion}

Several studies have evaluated sealer cytotoxicity using in vitro cell culture assay, implantation into muscle [3] and periradicular response [4]. In vivo tests are based on clinical and histological evaluation of tissue responses:

In the present study, materials were injected subcutaneously into the tissue in a controlled manner [5]. All sealers used in this study were aggressive to the subcutaneous tissue in the beginning of the experiment. The inflammatory reaction, however, become milder on the 30th day [6] .A stronger action of the sealers in the beginning and annulling of the inflammatory response over time have been reported elsewhere [7].

On the $1^{\text {st }}$ day, the reaction observed to all sealers was more likely due to the surgical trauma rather than caused by the materials' toxicity [6,7]. However, it allowed evaluating the behavior of the materials along the experimental time and during the natural skin healing process as the initial period [8, 9, and 10]. At this time, the tissue was disorganized and infiltrated with neutrophils, which is consistent with the findings of other studies $[11,12]$.

On the $5^{\text {th }}$ day, the tissue was disorganized and infiltrated with neutrophils, which is consistent with the findings of other studies[13].A combination of acute and chronic inflammatory cells were seen[14,15]. On the $10^{\text {th }}$ day, the tissue was more organized in all sealer groups and was infiltrated with chronic cells, such as macrophages, lymphocytes and plasma cells. Fibrous capsule formation was observed only with Diaket $[16,17,18]$ and Tubliseal[19,20,21].

On the 30th day, although the tissue inflammatory reaction to all sealers was milder than that observed on the $1^{\text {st }}$ day after injection, it was still present. Surrounding Tubliseal [22], a persistent inflammatory response was observed, which has already been reported. This could be attributed to eugenol release from this material whose eugenol content is high right after mixing, but decreases with time [24, 25]. Zinc-oxide-and-eugenolbased sealers have residual eugenol after mixing. As previously stated, this residual eugenol $(>5 \%)$ is sufficient to cause an inflammation. In the present study freshly mixed Diaket was placed directly into the tissue [26, 27].At the end of 30 days, histopathological observation exhibited chronic inflammatory reaction with mild amount of necrosis and foreign body reaction. The cytotoxicity did not decrease proportional to their setting time as does that of zinc oxide eugenol based sealer tested under the same parameter[28,29,]. Therefore the possibility that these type of sealers could irritate periapical tissue for a longer period of time should be considered [30,31]Inflammatory reactions associated with seal apex is in equal magnitude as that of other sealers in the initial stage $[32,33,34]$,but by around $10^{\text {th }}$ day inflammatory cells subsided drastically and a healing potential has become active by around 30 days[35,36].

In view of the methodological differences among in vivo investigations, it is difficult to compare directly our results to those of previous studies [37, 38]. Further research should be conducted to contribute to the development of a root canal sealer that fulfills all properties of an ideal material. 


\section{Figures And Tables}

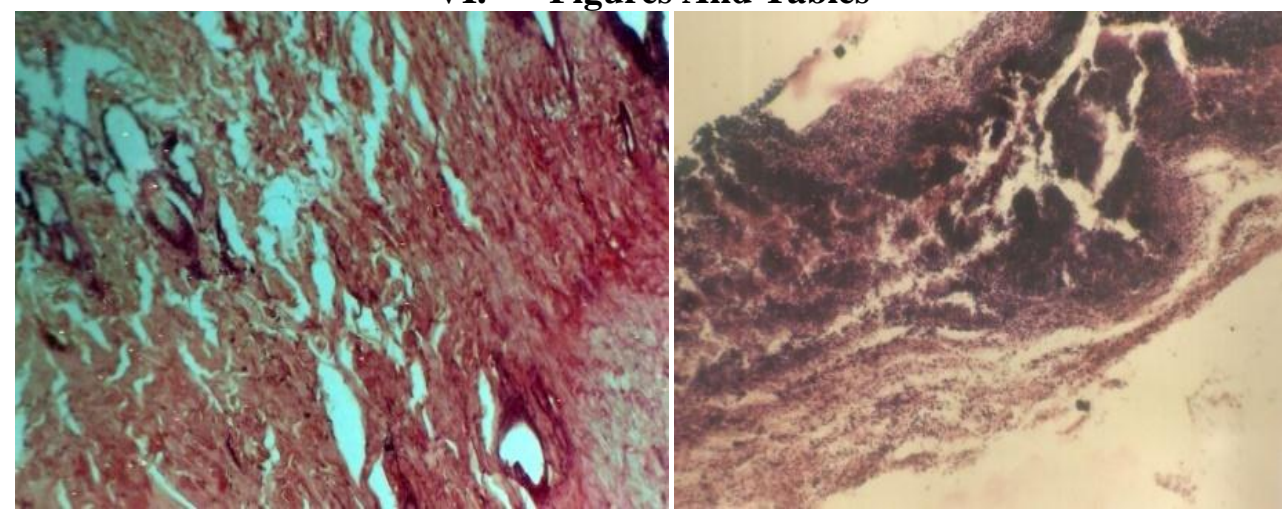

Fig $1 \mathrm{~A}$

Fig $1 \mathrm{~B}$
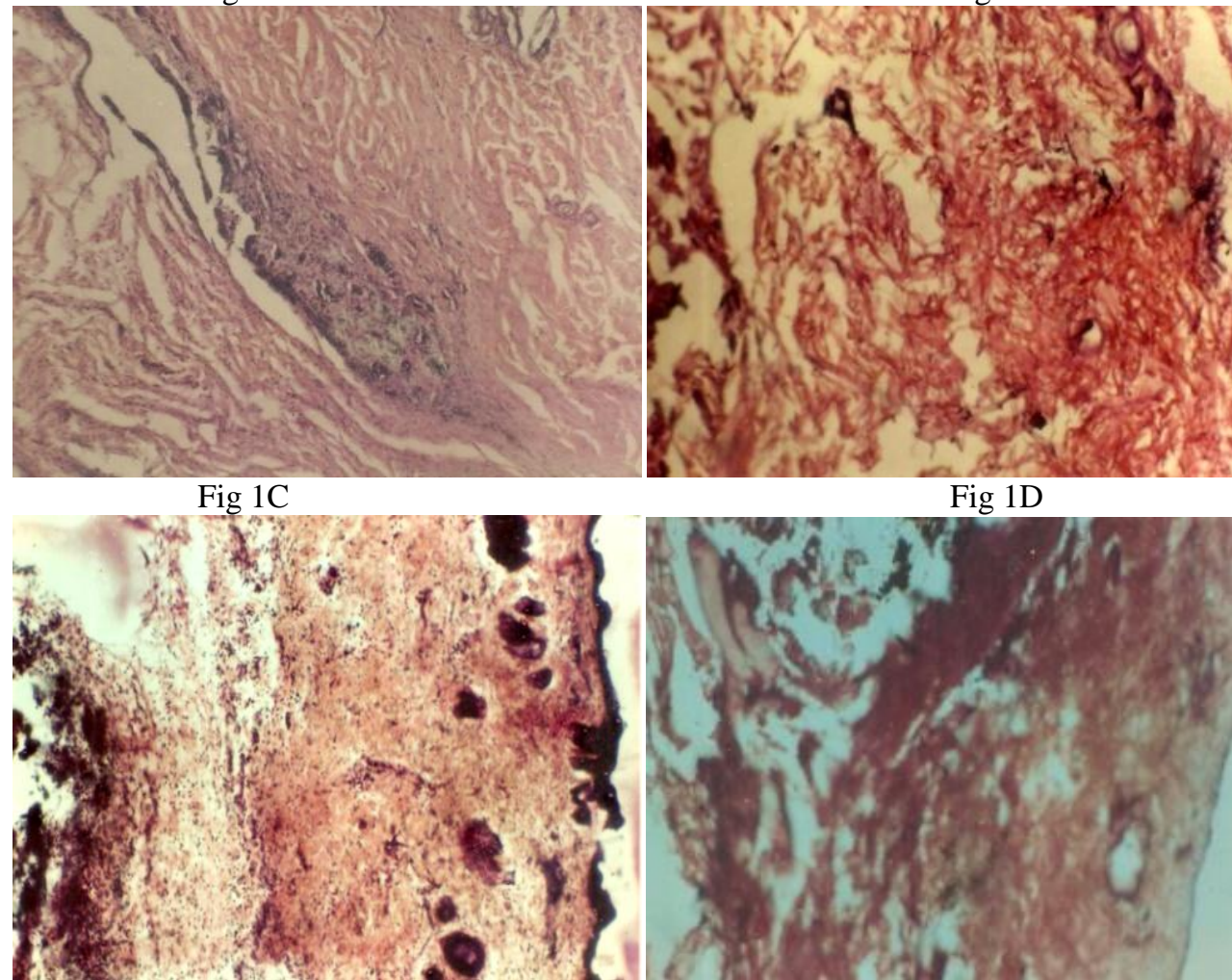

Fig 1D

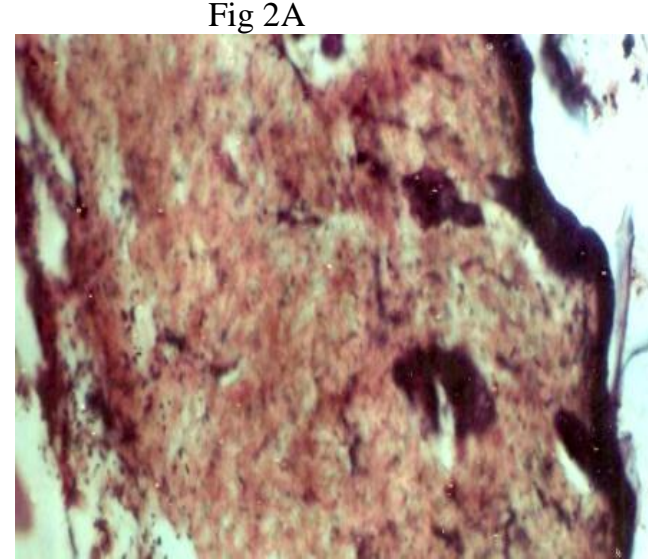

Fig $2 \mathrm{C}$

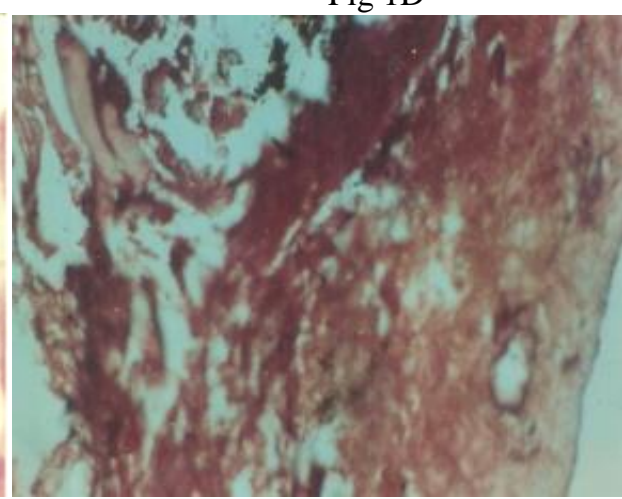

Fig 2B

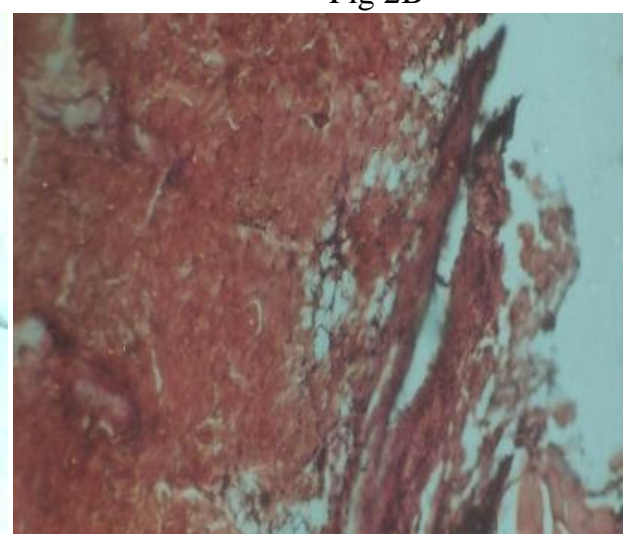

Fig 2D 


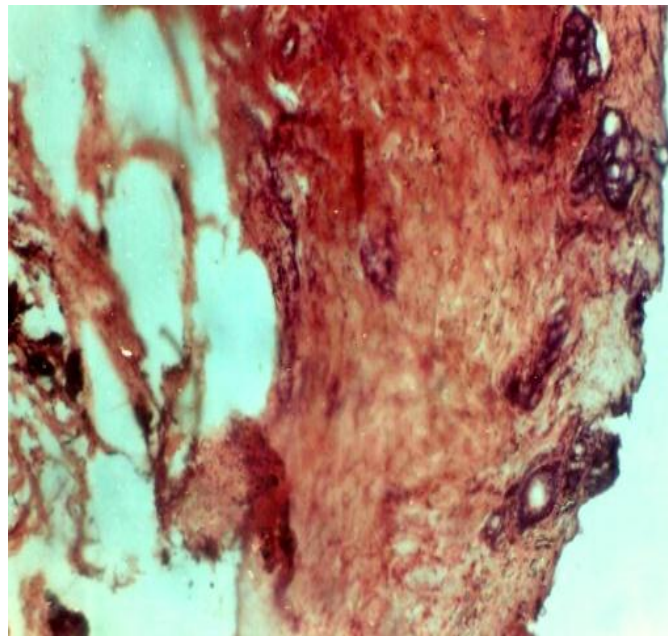

Fig $3 \mathrm{~A}$

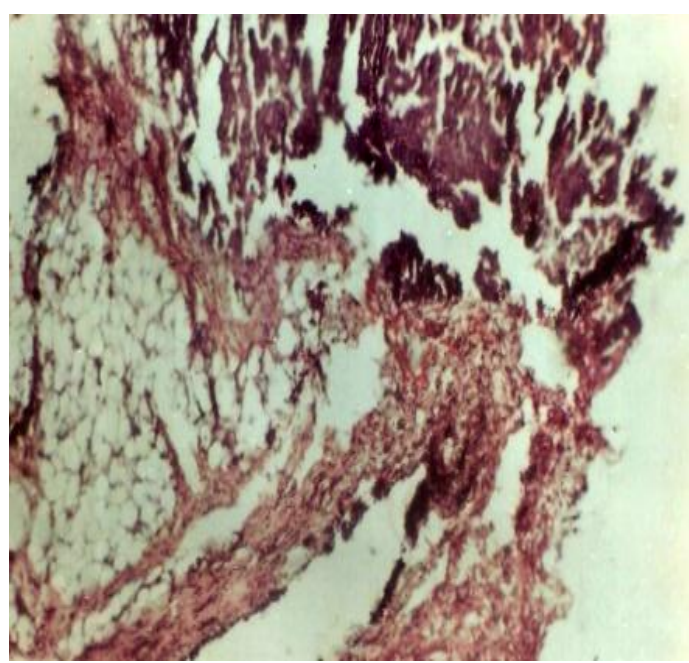

Fig 3C

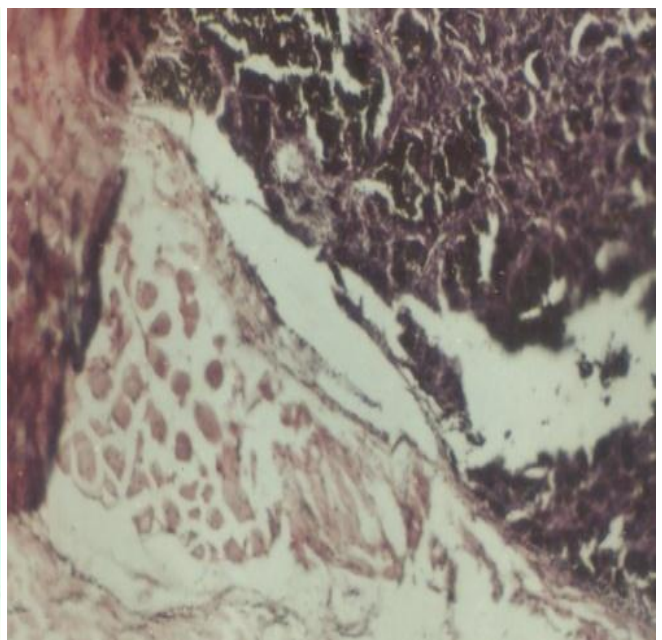

Fig 3B

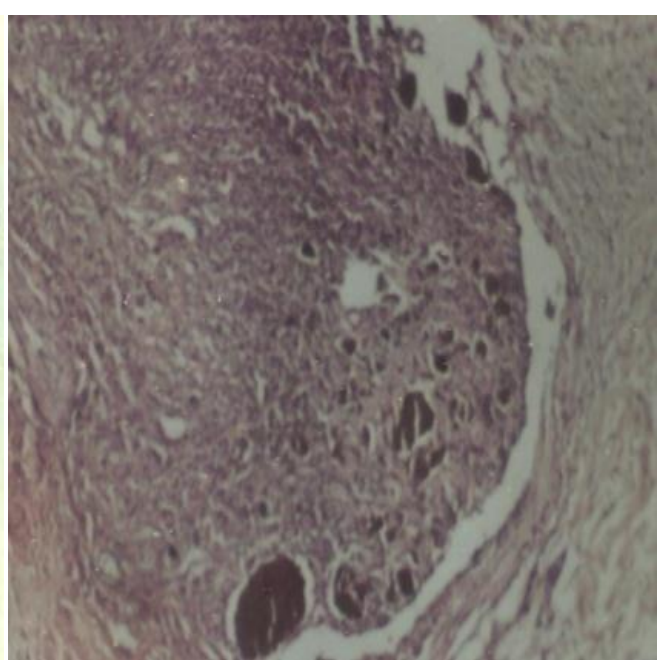

Fig3D

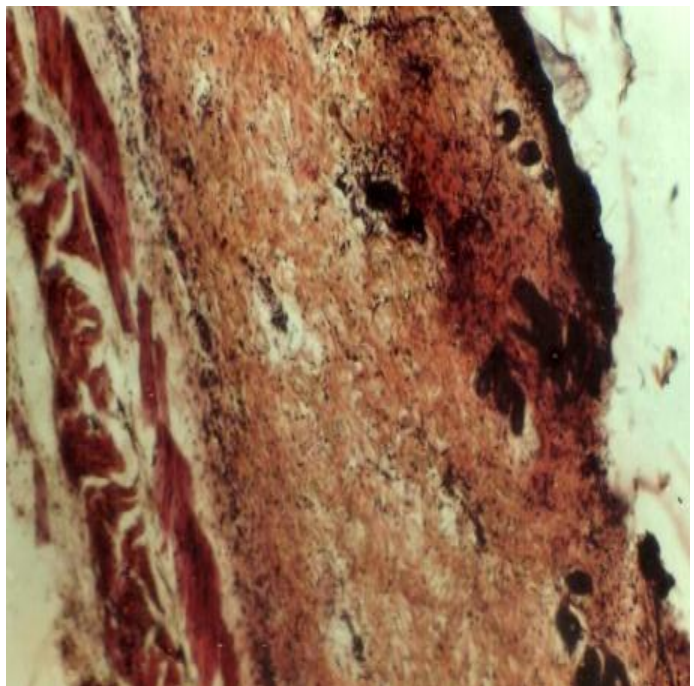

Fig 4A

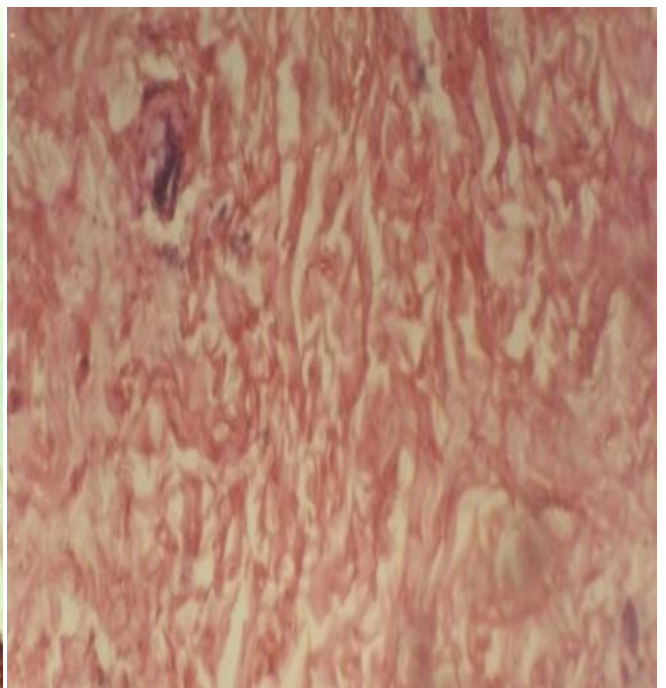

Fig 4D 
Histopathologic Evaluation of the Subcutaneous Tissue Response to Three Endodontic Sealers

TABLE.1 Comparison of acute inflammation among the material used at day1

\begin{tabular}{|c|c|c|c|c|c|c|c|}
\hline Inflamation & Severity & Sealapex & Diaket & Tubliseal & $\begin{array}{l}\text { Distilled } \\
\text { water }\end{array}$ & Z\# & $\mathrm{p}$ \\
\hline \multirow{5}{*}{ PMNLs } & Nil & 1 & 1 & 1 & 1 & \multirow{5}{*}{3.59} & \multirow{5}{*}{0.166} \\
\hline & Mild & 0 & 3 & 1 & 4 & & \\
\hline & Moderate & 1 & 1 & 3 & 0 & & \\
\hline & Severe & 3 & 0 & 0 & 0 & & \\
\hline & Mean \pm SD & $2.2 \pm 1.3$ & $1 \pm 0.71$ & $1.4 \pm 0.89$ & $0.8 \pm 0.45$ & & \\
\hline \multirow{4}{*}{ Eosinophils } & Nil & 1 & 4 & 4 & 5 & \multirow{4}{*}{5.12} & \multirow{4}{*}{0.077} \\
\hline & Mild & 3 & 1 & 1 & 0 & & \\
\hline & Moderate & 1 & 0 & 0 & 0 & & \\
\hline & Mean \pm SD & $1 \pm 0.71$ & $0.2 \pm 0.45$ & $0.2 \pm 0.45$ & $0 \pm 0$ & & \\
\hline \multirow{4}{*}{ lymphocytes } & Nil & 0 & 4 & 5 & 4 & \multirow{4}{*}{$10.91 * *$} & \multirow{4}{*}{0.004} \\
\hline & Mild & 4 & 1 & 0 & 1 & & \\
\hline & Moderate & 1 & 0 & 0 & 0 & & \\
\hline & Mean \pm SD & $1.2 \pm 0.45$ & $0.2 \pm 0.45$ & $0 \pm 0$ & $0.2 \pm 0.45$ & & \\
\hline
\end{tabular}

\# Kruskal Wallis Test

Lymphocytes : SealapexvsDiaket

SealapexvsTubliseal

Sealapexvs Distilled water
$\mathrm{Z}=2.4, \mathrm{p}<0.05 \quad$ (Mann-Whitney $\mathrm{U}$ test $)$

$\mathrm{Z}=2.9, \mathrm{p}<0.01$

$\mathrm{Z}=2.4, \mathrm{p}<0.05$

TABLE 2. Comparison of chronic inflammation among the material used at day1

\begin{tabular}{|c|c|c|c|c|c|c|c|}
\hline Inflamation & Severity & Sealapex & Diaket & Tubliseal & $\begin{array}{l}\text { Distilled } \\
\text { water }\end{array}$ & Z\# & $\mathrm{p}$ \\
\hline \multirow{3}{*}{ Plasma cell } & Nil & 5 & 4 & 4 & 5 & \multirow{3}{*}{1.08} & \multirow{3}{*}{0.584} \\
\hline & Mild & 0 & 1 & 1 & 0 & & \\
\hline & Mean \pm SD & $0 \pm 0$ & $0.2 \pm 0.45$ & $0.2 \pm 0.45$ & $0 \pm 0$ & & \\
\hline \multirow{3}{*}{ Histiocytes } & Nil & 4 & 4 & 5 & 4 & \multirow{3}{*}{1.08} & \multirow{3}{*}{0.584} \\
\hline & Mild & 1 & 1 & 0 & 1 & & \\
\hline & Mean \pm SD & $0.2 \pm 0.45$ & $0.2 \pm 0.45$ & $0 \pm 0$ & $0.2 \pm 0.45$ & & \\
\hline \multirow{3}{*}{ Giant cells } & Nil & 4 & 4 & 5 & 4 & \multirow{3}{*}{1.08} & \multirow{3}{*}{0.584} \\
\hline & Mild & 1 & 1 & 0 & 1 & & \\
\hline & Mean \pm SD & $0.2 \pm 0.45$ & $0.2 \pm 0.45$ & $0 \pm 0$ & $0.2 \pm 0.45$ & & \\
\hline \multirow{4}{*}{ Oedema } & Nil & 5 & 2 & 1 & 5 & \multirow{4}{*}{$7.22 *$} & \multirow{4}{*}{0.027} \\
\hline & Mild & 0 & 3 & 1 & 0 & & \\
\hline & Moderate & 0 & 0 & 3 & 0 & & \\
\hline & Mean \pm SD & $0 \pm 0$ & $0.6 \pm 0.55$ & $1.4 \pm 0.89$ & $0 \pm 0$ & & \\
\hline \multirow{3}{*}{$\begin{array}{l}\text { Granulation } \\
\text { tissue }\end{array}$} & Nil & 4 & 4 & 4 & 4 & \multirow{3}{*}{0} & \multirow{3}{*}{1.000} \\
\hline & Mild & 1 & 1 & 1 & 1 & & \\
\hline & Mean \pm SD & $0.2 \pm 0.45$ & $0.2 \pm 0.45$ & $0.2 \pm 0.45$ & $0.2 \pm 0.45$ & & \\
\hline \multirow{3}{*}{$\begin{array}{l}\text { Muscle } \\
\text { necrosis }\end{array}$} & Nil & 4 & 4 & 4 & 4 & \multirow{3}{*}{0} & \multirow{3}{*}{1.000} \\
\hline & Mild & 1 & 1 & 1 & 1 & & \\
\hline & Mean \pm SD & $0.2 \pm 0.45$ & $0.2 \pm 0.45$ & $0.2 \pm 0.45$ & $0.2 \pm 0.45$ & & \\
\hline \multirow{3}{*}{ Fibrosis } & Nil & 4 & 5 & 4 & 4 & \multirow{3}{*}{1.08} & \multirow{3}{*}{0.584} \\
\hline & Mild & 1 & 0 & 1 & 1 & & \\
\hline & Mean \pm SD & $0.2 \pm 0.45$ & $0 \pm 0$ & $0.2 \pm 0.45$ & $0.2 \pm 0.45$ & & \\
\hline \multirow{3}{*}{$\begin{array}{l}\text { Foreign body } \\
\text { reaction }\end{array}$} & Nil & 4 & 4 & 4 & 5 & \multirow{3}{*}{0} & \multirow{3}{*}{1.000} \\
\hline & Mild & 1 & 1 & 1 & 0 & & \\
\hline & Mean \pm SD & $0.2 \pm 0.45$ & $0.2 \pm 0.45$ & $0.2 \pm 0.45$ & $0 \pm 0$ & & \\
\hline
\end{tabular}

\# Kruskal Wallis Test

TABLE 3 Comparison of acute inflammation among the material used at day 5

\begin{tabular}{|c|c|c|c|c|c|c|c|}
\hline Inflamation & Severity & Sealapex & Diaket & Tubliseal & $\begin{array}{l}\text { Distilled } \\
\text { water }\end{array}$ & Z\# & $\mathrm{p}$ \\
\hline \multirow{5}{*}{ PMNLs } & Nil & 1 & 1 & 1 & 4 & \multirow{5}{*}{4.36} & \multirow{5}{*}{0.113} \\
\hline & Mild & 0 & 4 & 1 & 1 & & \\
\hline & Moderate & 1 & 0 & 3 & 0 & & \\
\hline & Severe & 3 & 0 & 0 & 0 & & \\
\hline & Mean \pm SD & $2.2 \pm 1.3$ & $0.8 \pm 0.45$ & $1.4 \pm 0.89$ & $0.2 \pm 0.45$ & & \\
\hline \multirow{3}{*}{ Eosinophils } & Nil & 5 & 4 & 5 & 4 & \multirow{3}{*}{2} & \multirow{3}{*}{0.368} \\
\hline & Mild & 0 & 1 & 0 & 1 & & \\
\hline & Mean \pm SD & $0 \pm 0$ & $0.2 \pm 0.45$ & $0 \pm 0$ & $0.2 \pm 0.45$ & & \\
\hline \multirow{3}{*}{ Lymphocytes } & Nil & 1 & 1 & 1 & 5 & \multirow{3}{*}{0} & \multirow{3}{*}{1.000} \\
\hline & Mild & 4 & 4 & 4 & 0 & & \\
\hline & Mean \pm SD & $0.8 \pm 0.45$ & $0.8 \pm 0.45$ & $0.8 \pm 0.45$ & $0 \pm 0$ & & \\
\hline
\end{tabular}

\# Kruskal Wallis Test 
Histopathologic Evaluation of the Subcutaneous Tissue Response to Three Endodontic Sealers

TABLE 4 Comparison of acute and chronic inflammation among the material used at day 5

\begin{tabular}{|c|c|c|c|c|c|c|c|}
\hline Inflamation & Severity & Sealapex & Diaket & Tubliseal & $\begin{array}{l}\text { Distilled } \\
\text { water }\end{array}$ & Z\# & $\mathrm{p}$ \\
\hline Plasma cell & Nil & 5 & 5 & 5 & 5 & 0 & 1.000 \\
\hline \multirow{3}{*}{ Histiocytes } & Nil & 4 & 4 & 5 & 4 & \multirow{3}{*}{1.08} & \multirow{3}{*}{0.584} \\
\hline & Mild & 1 & 1 & 0 & 1 & & \\
\hline & Mean \pm SD & $0.2 \pm 0.45$ & $0.2 \pm 0.45$ & $0 \pm 0$ & $0.2 \pm 0.45$ & & \\
\hline \multirow{3}{*}{ Giant cells } & Nil & 5 & 4 & 4 & 4 & \multirow{3}{*}{1.08} & \multirow{3}{*}{0.584} \\
\hline & Mild & 0 & 1 & 1 & 1 & & \\
\hline & Mean \pm SD & $0 \pm 0$ & $0.2 \pm 0.45$ & $0.2 \pm 0.45$ & $0.2 \pm 0.45$ & & \\
\hline \multirow{3}{*}{ Oedema } & Nil & 4 & 1 & 5 & 4 & \multirow{3}{*}{$7.28^{*}$} & \multirow{3}{*}{0.026} \\
\hline & Mild & 1 & 4 & 0 & 1 & & \\
\hline & Mean \pm SD & $0.2 \pm 0.45$ & $0.8 \pm 0.45$ & $0 \pm 0$ & $0.2 \pm 0.45$ & & \\
\hline \multirow{3}{*}{$\begin{array}{l}\text { Granulation } \\
\text { tissue }\end{array}$} & Nil & 4 & 4 & 4 & 5 & \multirow{3}{*}{0} & \multirow{3}{*}{1.000} \\
\hline & Mild & 1 & 1 & 1 & 0 & & \\
\hline & Mean \pm SD & $0.2 \pm 0.45$ & $0.2 \pm 0.45$ & $0.2 \pm 0.45$ & $0 \pm 0$ & & \\
\hline \multirow{3}{*}{ Muscle necrosis } & Nil & 1 & 1 & 1 & 5 & \multirow{3}{*}{0} & \multirow{3}{*}{1.000} \\
\hline & Mild & 4 & 4 & 4 & 0 & & \\
\hline & Mean \pm SD & $0.8 \pm 0.45$ & $0.8 \pm 0.45$ & $0.8 \pm 0.45$ & $0 \pm 0$ & & \\
\hline \multirow{4}{*}{ Fibrosis } & Nil & 4 & 2 & 1 & 4 & \multirow{4}{*}{5.32} & \multirow{4}{*}{0.070} \\
\hline & Mild & 1 & 3 & 1 & 1 & & \\
\hline & Moderate & 0 & 0 & 3 & 0 & & \\
\hline & Mean \pm SD & $0.2 \pm 0.45$ & $0.6 \pm 0.55$ & $1.4 \pm 0.89$ & $0.2 \pm 0.45$ & & \\
\hline \multirow{4}{*}{$\begin{array}{l}\text { Foreign } \\
\text { reaction }\end{array}$} & Nil & 4 & 4 & 4 & 4 & \multirow{4}{*}{0} & \multirow{4}{*}{1.000} \\
\hline & Mild & 1 & 1 & 1 & 0 & & \\
\hline & Moderate & 0 & 0 & 0 & 1 & & \\
\hline & Mean \pm SD & $0.2 \pm 0.45$ & $0.2 \pm 0.45$ & $0.2 \pm 0.45$ & $0.4 \pm 0.89$ & & \\
\hline
\end{tabular}

\# Kruskal Wallis Test

Oedema :DiaketVsTubliseal $\quad \mathrm{Z}=2.5, \mathrm{p}<0.05$

TABLE 5 Comparison of acute inflammation among the material used at day 10

\begin{tabular}{|c|c|c|c|c|c|c|c|}
\hline Inflamation & Severity & Sealapex & Diaket & Tubliseal & $\begin{array}{l}\text { Distilled } \\
\text { water }\end{array}$ & Z\# & $\mathrm{p}$ \\
\hline \multirow{3}{*}{ PMNLs } & Nil & 4 & 4 & 3 & 4 & \multirow{3}{*}{0.64} & \multirow{3}{*}{0.727} \\
\hline & Mild & 1 & 1 & 2 & 1 & & \\
\hline & Mean \pm SD & $0.2 \pm 0.45$ & $0.2 \pm 0.45$ & $0.4 \pm 0.55$ & $0.2 \pm 0.45$ & & \\
\hline \multirow{3}{*}{ Eosinophils } & Nil & 4 & 4 & 4 & 5 & \multirow{3}{*}{0} & \multirow{3}{*}{1.000} \\
\hline & Mild & 1 & 1 & 1 & 0 & & \\
\hline & Mean \pm SD & $0.2 \pm 0.45$ & $0.2 \pm 0.45$ & $0.2 \pm 0.45$ & $0 \pm 0$ & & \\
\hline \multirow{5}{*}{ Lymphocytes } & Nil & 1 & 0 & 1 & 4 & \multirow{5}{*}{$8.47^{*}$} & \multirow{5}{*}{0.014} \\
\hline & Mild & 0 & 0 & 1 & 1 & & \\
\hline & Moderate & 4 & 1 & 3 & 0 & & \\
\hline & Severe & 0 & 4 & 0 & 0 & & \\
\hline & Mean \pm SD & $1.6 \pm 0.89$ & $2.8 \pm 0.45$ & $1.4 \pm 0.89$ & $0.2 \pm 0.45$ & & \\
\hline
\end{tabular}

\# Kruskal Wallis Test

Lymphocytes : SealapexvsDiaket $\mathrm{Z}=2.4, \mathrm{p}<0.05$

Sealapexvs Distilled water $\quad \mathrm{Z}=2.2, \mathrm{p}<0.01$

DiaketVsTubliseal $\quad \mathrm{Z}=2.5, \mathrm{p}<0.05$

DiaketVs Distilled wate $\quad \mathrm{Z}=2.8, \mathrm{p}<0.01$

TublisealVs Distilled wate $Z=2.0, \mathrm{p}<0.05$

TABLE 6 Comparison of acute and chronic inflammation among the material used at day 10

\begin{tabular}{|c|c|c|c|c|c|c|c|}
\hline Inflamation & Severity & Sealapex & Diaket & Tubliseal & $\begin{array}{l}\text { Distilled } \\
\text { water }\end{array}$ & Z\# & $\mathrm{p}$ \\
\hline \multirow{4}{*}{ Plasma cell } & Nil & 1 & 1 & 2 & 5 & \multirow{4}{*}{1.14} & \multirow{4}{*}{0.565} \\
\hline & Mild & 3 & 4 & 3 & 0 & & \\
\hline & Moderate & 1 & 0 & 0 & 0 & & \\
\hline & Mean \pm SD & $1 \pm 0.71$ & $0.8 \pm 0.45$ & $0.6 \pm 0.55$ & $0 \pm 0$ & & \\
\hline \multirow{3}{*}{ Histiocytes } & Nil & 1 & 1 & 1 & 4 & \multirow{3}{*}{0} & \multirow{3}{*}{1.000} \\
\hline & Mild & 4 & 4 & 4 & 1 & & \\
\hline & Mean \pm SD & $0.8 \pm 0.45$ & $0.8 \pm 0.45$ & $0.8 \pm 0.45$ & $0.2 \pm 0.45$ & & \\
\hline \multirow{3}{*}{ Giant cells } & Nil & 2 & 1 & 1 & 5 & \multirow{3}{*}{0.64} & \multirow{3}{*}{0.727} \\
\hline & Mild & 3 & 4 & 4 & 0 & & \\
\hline & Mean \pm SD & $0.6 \pm 0.55$ & $0.8 \pm 0.45$ & $0.8 \pm 0.45$ & $0 \pm 0$ & & \\
\hline \multirow{4}{*}{ Oedema } & Nil & 5 & 0 & 0 & 4 & \multirow{4}{*}{$11.54 * *$} & \multirow{4}{*}{0.003} \\
\hline & Mild & 0 & 4 & 4 & 1 & & \\
\hline & Moderate & 0 & 1 & 1 & 0 & & \\
\hline & Mean \pm SD & $0 \pm 0$ & $1.2 \pm 0.45$ & $1.2 \pm 0.45$ & $0.2 \pm 0.45$ & & \\
\hline
\end{tabular}


Histopathologic Evaluation of the Subcutaneous Tissue Response to Three Endodontic Sealers

\begin{tabular}{|c|c|c|c|c|c|c|c|}
\hline \multirow{4}{*}{$\begin{array}{l}\text { Granulation } \\
\text { tissue }\end{array}$} & Nil & 0 & 1 & 1 & 1 & \multirow{4}{*}{0.78} & \multirow{4}{*}{0.679} \\
\hline & Mild & 1 & 1 & 1 & 4 & & \\
\hline & Moderate & 4 & 3 & 3 & 0 & & \\
\hline & Mean \pm SD & $1.8 \pm 0.45$ & $1.4 \pm 0.89$ & $1.4 \pm 0.89$ & $0.8 \pm 0.45$ & & \\
\hline \multirow{4}{*}{ Muscle necrosis } & Nil & 0 & 1 & 1 & 4 & \multirow{4}{*}{$8.63^{*}$} & \multirow{4}{*}{0.013} \\
\hline & Mild & 1 & 4 & 4 & 1 & & \\
\hline & Moderate & 4 & 0 & 0 & 0 & & \\
\hline & Mean \pm SD & $1.8 \pm 0.45$ & $0.8 \pm 0.45$ & $0.8 \pm 0.45$ & $0.2 \pm 0.45$ & & \\
\hline \multirow{3}{*}{ Fibrosis } & Nil & 4 & 1 & 2 & 4 & \multirow{3}{*}{3.5} & \multirow{3}{*}{0.174} \\
\hline & Mild & 1 & 4 & 3 & 1 & & \\
\hline & Mean \pm SD & $0.2 \pm 0.45$ & $0.8 \pm 0.45$ & $0.6 \pm 0.55$ & $0.2 \pm 0.45$ & & \\
\hline \multirow{4}{*}{$\begin{array}{l}\text { Foreign } \\
\text { reaction }\end{array}$} & Nil & 0 & 0 & 0 & 5 & \multirow{4}{*}{0} & \multirow{4}{*}{1.000} \\
\hline & Mild & 4 & 4 & 4 & 0 & & \\
\hline & Moderate & 1 & 1 & 1 & 0 & & \\
\hline & Mean \pm SD & $1.2 \pm 0.45$ & $1.2 \pm 0.45$ & $1.2 \pm 0.45$ & $0 \pm 0$ & & \\
\hline
\end{tabular}

\# Kruskal Wallis Test

Oedema

Muscle necrosis :
Sealapex vs Diaket

Sealapex vs Tubliseal

Diaket Vs Tubliseal

Tubliseal Vs Distilled water

Sealapex vs Diaket

Sealapex vs Tubliseal

Sealapex vs Distilled water
$\mathrm{Z}=2.9, \mathrm{p}<0.01$
$\mathrm{Z}=2.9, \mathrm{p}<0.01$
$\mathrm{Z}=2.4, \mathrm{p}<0.05$
$\mathrm{Z}=2.4, \mathrm{p}<0.05$
$\mathrm{Z}=2.4, \mathrm{p}<0.05$
$\mathrm{Z}=2.4, \mathrm{p}<0.05$
$\mathrm{Z}=2.7, \mathrm{p}<0.01$

TABLE 7 Comparison of acute inflammation among the material used at day 30

\begin{tabular}{|c|c|c|c|c|c|c|c|}
\hline Inflamation & Severity & Sealapex & Diaket & Tubliseal & $\begin{array}{l}\text { Distilled } \\
\text { water }\end{array}$ & Z\# & $\mathrm{p}$ \\
\hline \multirow{3}{*}{ PMNLs } & Nil & 4 & 4 & 4 & 5 & \multirow{3}{*}{0} & \multirow{3}{*}{1.000} \\
\hline & Mild & 1 & 1 & 1 & 0 & & \\
\hline & Mean \pm SD & $0.2 \pm 0.45$ & $0.2 \pm 0.45$ & $0.2 \pm 0.45$ & $0 \pm 0$ & & \\
\hline \multirow{3}{*}{ Eosinophils } & Nil & 5 & 3 & 5 & 4 & \multirow{3}{*}{4.31} & \multirow{3}{*}{0.116} \\
\hline & Mild & 0 & 2 & 0 & 1 & & \\
\hline & Mean \pm SD & $0 \pm 0$ & $0.4 \pm 0.55$ & $0 \pm 0$ & $0.2 \pm 0.45$ & & \\
\hline \multirow{5}{*}{ Lymphocytes } & Nil & 1 & 0 & 1 & 5 & \multirow{5}{*}{3.65} & \multirow{5}{*}{0.161} \\
\hline & Mild & 1 & 1 & 0 & 0 & & \\
\hline & Moderate & 3 & 1 & 4 & 0 & & \\
\hline & Severe & 0 & 3 & 0 & 0 & & \\
\hline & Mean \pm SD & $1.4 \pm 0.89$ & $2.4 \pm 0.89$ & $1.6 \pm 0.89$ & $0 \pm 0$ & & \\
\hline
\end{tabular}

\# Kruskal Wallis Test

Table Comparison of acute and chronic inflammation among the material used at day 30

\begin{tabular}{|c|c|c|c|c|c|c|c|}
\hline Inflamation & Severity & Sealapex & Diaket & Tubliseal & $\begin{array}{l}\text { Distilled } \\
\text { water }\end{array}$ & Z\# & $\mathrm{p}$ \\
\hline \multirow{3}{*}{ Plasma cell } & Nil & 1 & 1 & 2 & 4 & \multirow{3}{*}{0.64} & \multirow{3}{*}{0.727} \\
\hline & Mild & 4 & 4 & 3 & 1 & & \\
\hline & Mean \pm SD & $0.8 \pm 0.45$ & $0.8 \pm 0.45$ & $0.6 \pm 0.55$ & $0.2 \pm 0.45$ & & \\
\hline \multirow{3}{*}{ Histiocytes } & Nil & 1 & 2 & 1 & 5 & \multirow{3}{*}{0.64} & \multirow{3}{*}{0.727} \\
\hline & Mild & 4 & 3 & 4 & 0 & & \\
\hline & Mean \pm SD & $0.8 \pm 0.45$ & $0.6 \pm 0.55$ & $0.8 \pm 0.45$ & $0 \pm 0$ & & \\
\hline \multirow{3}{*}{ Giant cells } & Nil & 1 & 1 & 1 & 4 & \multirow{3}{*}{0} & \multirow{3}{*}{1.000} \\
\hline & Mild & 4 & 4 & 4 & 1 & & \\
\hline & Mean \pm SD & $0.8 \pm 0.45$ & $0.8 \pm 0.45$ & $0.8 \pm 0.45$ & $0.2 \pm 0.45$ & & \\
\hline \multirow{3}{*}{ Oedema } & Nil & 5 & 5 & 5 & 4 & \multirow{3}{*}{0} & \multirow{3}{*}{1.000} \\
\hline & Mild & 0 & 0 & 0 & 1 & & \\
\hline & Mean \pm SD & $0 \pm 0$ & $0 \pm 0$ & $0 \pm 0$ & $0.2 \pm 0.45$ & & \\
\hline \multirow{3}{*}{$\begin{array}{l}\text { Granulation } \\
\text { tissue }\end{array}$} & Nil & 4 & 4 & 4 & 1 & \multirow{3}{*}{0} & \multirow{3}{*}{1.000} \\
\hline & Mild & 1 & 1 & 1 & 4 & & \\
\hline & Mean \pm SD & $0.2 \pm 0.45$ & $0.2 \pm 0.45$ & $0.2 \pm 0.45$ & $0.8 \pm 0.45$ & & \\
\hline \multirow{3}{*}{ Muscle necrosis } & Nil & 5 & 1 & 1 & 5 & \multirow{3}{*}{$8^{*}$} & \multirow{3}{*}{0.018} \\
\hline & Mild & 0 & 4 & 4 & 0 & & \\
\hline & Mean $\pm \mathrm{SD}$ & $0 \pm 0$ & $0.8 \pm 0.45$ & $0.8 \pm 0.45$ & $0 \pm 0$ & & \\
\hline \multirow{4}{*}{ Fibrosis } & Nil & 1 & 0 & 1 & 1 & \multirow{4}{*}{5.55} & \multirow{4}{*}{0.062} \\
\hline & Mild & 4 & 1 & 1 & 4 & & \\
\hline & Moderate & 0 & 4 & 3 & 0 & & \\
\hline & Mean \pm SD & $0.8 \pm 0.45$ & $1.8 \pm 0.45$ & $1.4 \pm 0.89$ & $0.8 \pm 0.45$ & & \\
\hline \multirow{3}{*}{$\begin{array}{l}\text { Foreign body } \\
\text { reaction }\end{array}$} & Nil & 1 & 1 & 1 & 5 & \multirow{3}{*}{4.65} & \multirow{3}{*}{0.098} \\
\hline & Mild & 4 & 0 & 0 & 0 & & \\
\hline & Moderate & 0 & 4 & 4 & 0 & & \\
\hline
\end{tabular}


Histopathologic Evaluation of the Subcutaneous Tissue Response to Three Endodontic Sealers

\# Kruskal Wallis Test

\begin{tabular}{l|l|l|l|l}
\hline Mean \pm SD & $0.8 \pm 0.45$ & $1.6 \pm 0.89$ & $1.6 \pm 0.89$ & $0 \pm 0$ \\
\hline
\end{tabular}

Muscle necrosis :

$\begin{array}{lc}\text { SealapexvsDiaket } & \mathrm{Z}=2.5, \mathrm{p}<0.05 \\ \text { SealapexvsTubliseal } & \mathrm{Z}=2.5, \mathrm{p}<0.05 \\ \text { DiaketVs Distilled wate } & \mathrm{Z}=2.5, \mathrm{p}<0.05\end{array}$

DiaketVs Distilled wate $\mathrm{Z}=2.5, \mathrm{p}<0.05$

TublisealVs Distilled wate $\mathrm{Z}=2.5, \mathrm{p}<0.05$

\section{Conclusion}

Under the tested conditions, it may be concluded that the sealers had a similar pattern of irritation, which was more severe in the beginning and milder with time, in such a way that all sealers showed a persistent mild reaction. Sealapex yielded better tissue organization than Diaket and Tubliseal. Diaket showed a persistent chronic inflammation till a period of 30 days, while for. Tubliseal the tissue reaction became negligible at the end of study period.

\section{Acknowledgement}

I express my sincere gratitude and indebtedness to my most respected Professor Dr. B.Sureshchandra.My thesis work would never have been possible without his constant guidance and support.

I gratefully acknowledge the valuble guidance given by Dr. Khadilkar(pathologist) for analysis and interpretation of slides

I am thankful to Dr. Oommen P. Mathew, for the help rendered in analyzing the statistical aspect of my research.

\section{References}

[1]. Serge Bouillaguet,John.C.Watahaetal,Cytotoxicity and sealing properties of four classes of endodontic sealers evaluated by succinic acid dehydrogenase activity and confocal laser scanning microscopy, European Journal of Oral Sciences, 112(2),2004,182-87.

[2]. M Aminozarbian M D,E Balimani M D etal,Histopathological evaluation of subcutaneous tissue response to endodontic sealers,Dental Research journal ,2(2), 2006,64-7.

[3]. Huang T H ,Ding S H,Cytotoxicity of resin-zinc oxide eugenol and calcium hydroxide on pdl cells and permanent V79 cells, Int Endo J.35(2),2002,153-8.

[4]. Ingle J I.Ingles Endodontics. $6^{\text {th }}$ ed.Connecticut.Pmph USA ltd :2008

[5]. TorabenejiadM,Walton R E,Endodontics.Prnciples and Practice-Philadelphia,W B Saunders Co, 2009.

[6]. SanjeevTyagi ,PriyeshMishra,Evolution of root canal sealers:An insight story,European Journal of General Dentistry ,2(3),2013,199-218.

[7]. Weadl.M.Al-Omarietal,Survey of attitudes ,materials and methods employed in endodontic treatment by general Dental practicioners in North Jordan, B M C Health ,18(2) ,2004, 10-14.

[8]. Bun San Chong,Thomas R Pittford, Root end filling materials:rationale and tissue response, 11(1),2005,114-130.

[9]. PariciaSuzhuki,ValdirdeSouza,Roberto Holland etal,Tissue reaction to Endometasone sealer in root canal filling short or beyond the apical foramen,J.Appl.Oral Science,19(5), Sep/Oct2011.

[10]. D.E.Witherspoon,J L Gutman,Analysis of healing to guttapercha and Diaket when usead as aroot end filling materials in paeriapicalsurgeries, Int Endo Journa,l33(1),2000,37-45.

[11]. Man K Iqbal,RehaabSijing,Hishan Al Saidan,A comparison of sealing ability of four sealer cements in apically perforated root canals.Saudi Endodontic Journal,1(1),2011,12-18.

[12]. Callis P D,SatiniA,Tissue response to retrograde fillings in ferret canine: a comparison of glass ionomer cement and gutta-percha with sealers , J Triple O.64(4), 1987, 475-9.

[13]. VujaskovicMirjana,BaceticDragoljub,Tissue toxicity of root canal sealers,Serbian Dental journal,5(3),2004 ,136-141.

[14]. Grover R,MehraM,Clinical efficiency of varius root canal obturation methods in primary teeth: a comparative study,J of Pediatric Dentistry, 14(2),2013,104-8.

[15]. Tai K W,Huang F M,Assessment of genotoxicity of resin and zinc oxide eugenol based root canal sealers using in vitro mammalian test system,J Biomed Mater Res, 59(1),2002,73-5.

[16]. DomenioRicucci,Louis M Lin etal,Healing of apical tissue after root canal therapy,a long term clinical,radiographic and histopathologic observation study, Oral Radiology and Endodontology,10.8(4),2009, 609-201.

[17]. AshokanS ,SooryaPrakasC,Raghu, Volumetric analysis of root canal fillings in primary teeth using spiral computed tomography: an in vitro study,J Dental Child (chic), 79(2),2012, 46-8.

[18]. ZmenerO,Pameijir C H,Clinical and radiographic evaluation of resin based sealer-10 year recall data,Int J Dent 12,2012:763248.

[19]. Al Omari,Hammedetal,Reaction of rat connective tissue toMTA and Diaket,B M C Oral Health,11(17),2011,1472-6831.

[20]. Cornelis H Pameijier,OsvaldoZmener,Resin materials for root canal obturation.Dent Clinic North America ,54,2010,325-344.

[21]. FundaKontCobankara, HasanOrucoglu, Effectof five different resin based sealers on L929 and Saos -2 cell viability,J of Pediatric Dentistry, 1(2) 2013,37-41.

[22]. Zmener O, Pameijer $\mathrm{C}$ h,Subcutaneous connective tissue reaction to methacrylate resin based and zinc oxide based sealers,J Endo, 36(9), 2010,1574-9.

[23]. ParirokhM,MohammadianL,Comparison of subcutaneous reaction to three root canal sealers namely tubliseal,AH26,Dorofil,Journal of Dental School, 18(2), 2000,99-106.

[24]. ZmenerO,Pamejer C H,KobubuGA,Sub cutaneous connective tissue reactions to methacrylate resin based and zinc oxide eugenol based sealers,J of Endo, 36(9),2010, 1574-9.

[25]. Scarparo R K,Greca F S,Fachin E V,Analysis of tissue reactions to methacrylate resin based epoxy resin baed and zinc oxide eugenol based sealers,JEndodo,35(2), 2009,229-32.

[26]. Sandheep Thakur, DarshanaDevadiga, Resin based root canal sealers, An overview.Research Journal of Pharmaceutical,Biological and Chemical Sciences,4(4),2013, 1501-1509.

[27]. Ricardo AbrendaRosa,JulianaBrochetal,Influence of Endodontic sealer composition and time of fibre cementation in sealer adhesiveness to bovine root dentin, Brazilian dental Journal ,24(3), 2013, 241-246.

[28]. J.D.Regan,JGutman,Response of periradicular tissue to growth factors introduced into the surgical site as root end filling material,Int Endo Journal ,32(3),1999,171-182. 
[29]. Mittal M,ChandraS,Comparitive tissue toxicity evaluation of four sealers,J.Endo,21(12),1995,622-4.

[30]. Camps J, Pommel etal,Influence of powder liquid ratio on the properties of zinc oxide eugenol based root canal sealers,J Dent Material ,21(10),2004,915-23.

[31]. Huang F M,yang S ,Effect of root canal sealers on alkaline phosphatase inhuman osteoblasticcells,J Endo, 36(7),2010, 1230-3.

[32]. Elisagela de Fatima Rezende,Maria Christina etal,Evaluation of zinc oxide eugenol cement to Allium Cepa L.J of Biological Sciences, 35(4), 2013, 563-9.

[33]. AasthaPuri,SuchethaKumarietal, Value addition properties of zinc oxide eugenol after electron beam radiation,Research journal of Pharmaceutical,Biological and chemical science,4(2),2013, 243-6.

[34]. T Shimizu,TKawakami,TOchiai,Histopathological evaluation of subcutaneous tissue reaction in mice to calcium hydroxide paste developed for root canal fillings, J of International Medical research, 32(4), 2004, 416-21.

[35]. P Karnat,M K Manjunath,Roshini,Reaction of rat subcutaneous tissue to mineral trioxide and Portland cement.A secondary level biocompatibility test, J of Indian Soceity of Pedodontics and Preventive dentistry,31(2),2013, 74-81.

[36]. Marina Fernadez,RahulWagle,Toothresorption,part-1,pathogenesis and case series of internal resorption, 16(1) www.jcd 2013 4-8.

[37]. NurulAmeen-Inamdar,Salman H Mulla,Transportation of apical root canal after removal of Ca(OH) $)_{2}$ when used as an intracanalmedicament.An in vitro evaluation, 3(3),2013, 125-131 